\title{
A NEW CONCEPT IN CALCULATION OF THICK FILM RESISTORS
}

\author{
G. STECHER \\ Robert Bosch GmbH, Stuttgart, West Germany
}

(Received June 8, 1977)

\begin{abstract}
Because of interaction of resistor and conductor material and variation of film thickness due to different resistor geometries, square resistance becomes a function of resistor length and width. Additional to the square resistance one paste parameter or two parameters must be introduced if the influence of both length and width on the square resistance is to be taken into account. From resistance measurements of a test pattern all paste parameters can be calculated as numerical functions of resistor length by a computer programme using the method of least squares. Beside the layout of thick film resistors, the data are suitable to give a computer plot of square resistance in relation to length and width. This representation can play an important part in paste evaluation and process control. It shows at a glance what relationship will be present with various combinations of conductor and resistor materials or by changed process conditions.
\end{abstract}

\section{INTRODUCTION}

The layout of thickfilm resistors of small geometries would involve considerable systematic errors if the square resistance only is taken into account as a fixed paste parameter. Because of the dominant influence of resistor length, most studies have not treated the influence of resistor width on square resistance. ${ }^{2-6}$ In the cases in which this effect has been considered, ${ }^{7-10}$ no clear relationship which would be of use in designing a layout has been established.

In the method described here, it is shown that in addition to the square resistance, one paste parameter (No. 1 approximation) or two parameters (No. 2 approximation) must be introduced, if the influence of resistor width on the square resistance is to be taken into account.

All paste parameters can be rapidly determined, with the aid of a computer programme, as numerical functions of resistor length from resistance measurements on a test pattern. It is important to emphasize that the terms length $(L)$ and width $(W)$ of a resistor always mean the layout values, which can be taken into account only. (The dimensions on the substrate may be different to a certain extent, because of paste rheology etc., Figure 1).

The interaction of resistance material with the conductor connections will in most cases lower, but in some cases increase the square resistance of short resistors. In addition, the square resistance is influenced by the dependence of film thickness on
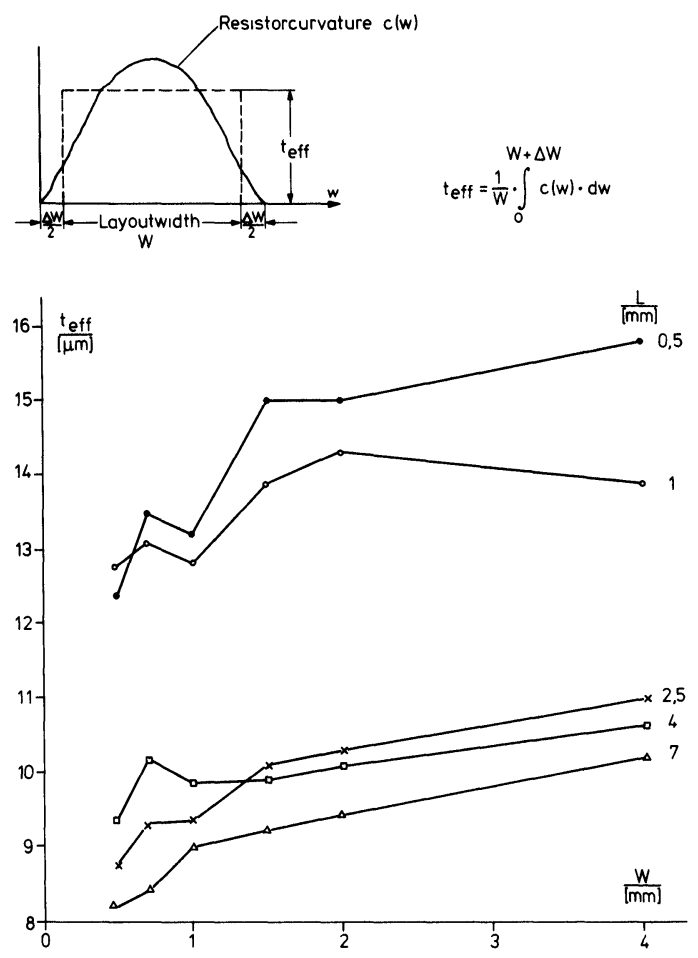

FIGURE 1 Effective thickness $t_{\text {eff }}$ of thick film resistors as a function of resistor width $W$ (Parameter: length $L$ ).

resistor geometry. The film thickness relations can be strongly influenced by rheological properties of 
resistor paste and by different screen printing parameters. Usually, however, short resistors show an increase in thickness, due to defective depression of the screen, while at the transition from a narrow to a broad resistor the film thickness likewise increases or forms a maximum. ${ }^{1}$

Figure 1 shows measurements of the film thickness as a function of resistor geometry.

\section{EXPERIMENTAL DATA}

In order to determine the paste parameters as numerical functions of $L$, a test pattern containing 36 resistors is used (Figure 2).

Each resistor length $L=0.5,1.0,1.5,2.5,4.0$ and $7.0 \mathrm{~mm}$, is present in widths $W=0.5,0.7,1.0$, $1.5,2.0$ and $4.0 \mathrm{~mm}$. To obtain statistical confidence limits for the values, 25 substrates with the same test pattern are used in each investigation. For the data, reported here, the conductor material is PdAg 9473 (Du Pont), pre-fired, thickness $13 \mu \mathrm{m}$. The resistor material is series 1300 (Du Pont), fired at a peak temperature of $860^{\circ} \mathrm{C}$ for $9 \mathrm{~min}$ to $10 \mathrm{~min}$. The resistor screen is stainless steel, 205 mesh (dia./ width $=50 / 75 \mu \mathrm{m}$ ), diagonally stretched, with an AZOCOL S direct mask 0-3 $\mu \mathrm{m}$ above the mesh. Squeegee movement is parallel to the long side of the test pattern.

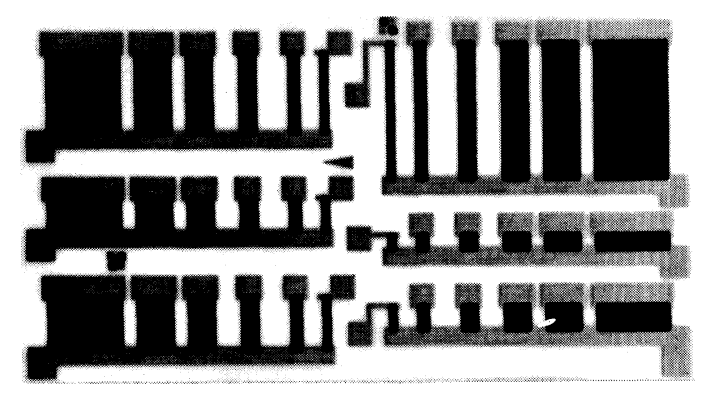

FIGURE 2 Resistor test pattern. Each resistor with a length $L=0.5,1,1.5,2.5,4,7 \mathrm{~mm}$ is present in widths $W=0.5,0.7,1,1.5,2,4 \mathrm{~mm}$.

\section{THE NEW RESISTANCE FORMULAS}

Because of the interaction of conductor material and resistor material and because of the dependence of film thickness on geometry, the square resistance and the effective thickness $t_{\text {eff }}$ are functions of the length and width of the resistor. The value of $t_{\mathrm{eff}}$ is the thickness which the actual resistor would have if its cross-section were rectangular and its width equal to the layout value $W$ (Figure 1). To separate the variables $L$ and $W$, we use a product formula:

$$
t_{\text {eff }}(L, W)=t_{\text {eff }}(L, 1) \cdot f(W)
$$

or by virtue of $\quad R^{\square} \sim 1 / t_{\text {eff }}$

$$
R^{\square}(L, W)=R^{\square}(L, 1) \cdot 1 / f(W)
$$

The first term in Eq. (1.1), a function of length only, is termed square resistance of the unit width. It is the first paste parameter and represents the curve of square resistance as a function of length but is now obtained for a definite width, namely the unit width $1 \mathrm{~mm}$. The unknown dimension less function $f(W)$, for which a formula must be found, describes the dependence of effective thickness in relation to width for a fixed length, according to Eq. (1). The first intention for $f(W)$ was to find a function which will describe the relationship between thickness and width in general, like Miller ${ }^{1}$ has found i.e. an increasing curve which may have a maximum (Figure 3 ). $\dagger$ Beside that, the function $f(W)$ must possess the following properties:

1) The function $f(W)$ must contain unknown parameters - the new paste parameters - which determine the form of $f(W)$ for constant $L$ so, that Eqs. (1) and (1.1) are satisfied. As the parameters are calculated for a fixed length from the 6 resistance means of the resistors with different widths, $t_{\text {eff }}$ in Eq. (1) represents the "electrically active thickness" as a function of $W$. This is a condition, transferred from Eq. (1) to Eq. (1.1).

2) The function $f(W)$ must have a form making possible the use of a suitable mathematical procedure for calculating the unknown parameters at constant $L$. Here $f(W)$ is chosen in such a way, that the method of least squares can be used.

3) By virtue of Eqs. (1) and (1.1), $f(W)$ must be equal to 1 when $W=1 \mathrm{~mm}$. The second requirement, in particular, leads to a function of the form

$$
f(W)=W^{m-1-a \cdot \ln W / 2}
$$

with the two new dimensionless parameters $a(L)$ and $m(L)$, which can be calculated as numerical functions of resistor length, as can the square resistance $R^{\square}(L, 1)$ also. The parameter $m(L)$ is termed the regression coefficient. It has numerical values in the neighbourhood of $1 . a(L)$ is here termed the parabolic coefficient. It has values of the order of \pm 0.05 . 


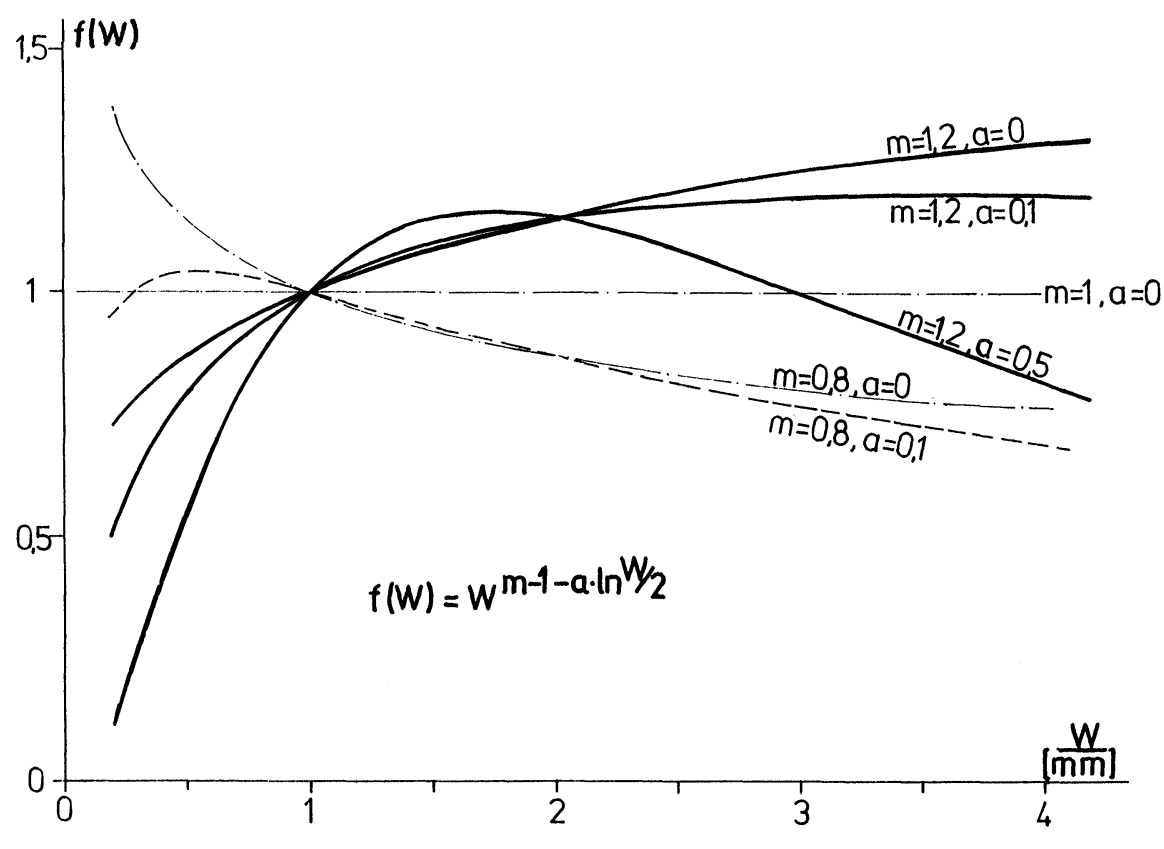

FIGURE 3 Some curves of the function $f(W)$.

Figure $3 \nmid$ shows the form of the function $f(W)$ for three different degrees of approximation. $m=1, a=0$ (zero approximation) and hence $f(W)$ $=1$. This is the trivial case: film thickness and square resistance are independent of $W$, by virtue of Eqs. (1) and (1.1).

$m \neq 1, a=0$ (first approximation) $f(W)$ is a root function if $m>1$ (e.g. in Figure 3, $m=1.2$ ).

$m \neq 1, a \neq 0$ (second approximation). The function $f(W)$ is now more flexible than in the first approximation and can describe a maximum in $t_{\text {eff }}(W)$. If $m<1$, the function $f(W)$ can also describe the case in which the effective film thickness $t_{\text {eff }}$ falls off with increasing width $W$. The reciprocal relationship gives the width dependence of square resistance. Its actual value is obtained when it is multiplied by the square resistance of the unit width $1 \mathrm{~mm}$, where the curves are always equal to one. The flexibility of the function $f(W)$, which is able to describe a increasing, a decreasing or a constant curve of $R^{\square}(L, W)$ as a function of width $W$, according to the measured resistance mean values, is a essential of the method. If Eqs. (2) and (1.1) are substituted in the equation

$\dagger$ The curves with $a \neq 0$ in Figure 3 which are published in the Proceedings of European Hybrid Microelectric Conference 1977, Bad Homburg, Germany, have been mistakenly drawn wrong.
$R=R^{\square}(L, W) \cdot L / W$, the new resistance equation is obtained. Zero approximation:

$$
R \approx R^{\square}(L, 1) \cdot L / W
$$

This is only valid if the numerical function $R^{\square}(L, \bar{W})$ for a "mean width $\bar{W}$ " is used in place of $R^{\square}(L, 1)$. First approximation:

$$
R=R_{1}^{\square}(L, 1) \cdot L \cdot W^{-m_{1}}
$$

Choosing a length, the width is calculated by

$$
W=\left(R_{1}^{\square}(L, 1) / R \cdot L\right)^{1 / m_{1}}
$$

Second approximation:

$$
R=R_{2}^{\square}(L, 1) \cdot L \cdot W^{a \cdot \ln W / 2-m_{2}}
$$

which gives for width $W$ the equation

$$
W=\exp \left\{\frac{m_{2}+a \cdot \ln 2}{2 a} \pm\right.
$$

$$
\left.\left[\frac{1}{a} \cdot \ln \frac{R}{R_{2}^{\square}(L, 1) \cdot L}+\left(\frac{m_{2}+a \cdot \ln 2}{2 a}\right)^{2}\right]^{1 / 2}\right\}
$$


(The negative sign being taken for $a>0$ and the positive for $a<0$ ).

\section{COMPUTATION OF THE PARAMETERS}

The computation of the paste parameters $R^{\square}(L, 1)$, $m(L)$ and $a(L)$ for each value of length $L$ in the test pattern is effected by a computer programme using the 36 measured mean values of resistances. The new resistance equations have such a form, that in a $x y$-coordinate system. with $x=\ln W$ and $y=\ln R$, the first approximation gives a straight line

$$
\begin{gathered}
\ln R=\ln \left(L \cdot R_{1}^{\square}(L, 1)\right)-m_{1} \cdot \ln W \\
y=c_{10}+c_{11} \cdot x
\end{gathered}
$$

and the second approximation a parabola

$$
\begin{aligned}
\ln R=\ln \left(L \cdot R_{2}^{\square}(L, 1)\right)- & \\
y= & c_{20}+ \\
& \left(a \cdot \ln 2+m_{2}\right) \cdot \ln W+a \cdot(\ln W)^{2} \\
& c_{21} \cdot x+c_{22} \cdot x^{2}
\end{aligned}
$$

for each of the 6 lengths of the test pattern.

The programme thus computes the 6 regression lines or 6 regression parabolas, for which the sums of squared deviations is a minimum. By means of the coefficients $c_{10}$ and $c_{11}$ the paste parameters of the first approximation $R_{1}(L, 1), m_{1}(L)$, and, with the coefficients $c_{20}, c_{21}$ and $c_{22}$, the parameters of the second approximation $R_{2}^{\square}(L, 1) m_{2}(L)$ and $a(L)$ can be determined. These are joined together to form polygons over the length $L$ (linear interpolation). In Table I, the corresponding values for the pastes 1311,1331 and 1361 in the second approximation are given.

\section{ACCURACY OF THE METHOD}

Figure 4 shows the distribution of errors between measured mean resistance values and values calculated from Eqs. (4) or (5), for all 6 pastes of the Du Pont 1300 series. In each case the 36 resistance geometries from $0.5 \times 0.5 \mathrm{~mm}^{2}$ to $7.0 \times 4.0 \mathrm{~mm}^{2}$ of the test pattern of Figure 2 are given. Each histogram thus gives the corresponding percentage deviations for $6 \times 36=216$ resistance mean values. The systematic error still perceptible in the first approximation is caused by resistors of different widths $W$. This error does not appear with the second approximation. The method has also been tried out with other paste systems - thick films, resinates and carbon pastes giving more or less the same distributions. Because method of least squares is used the mean error is equal to zero. Conditions in an actual circuit layout will unfortunately be different. The position of the resistors on the substrate may have some influence, the unevenness of the substrate surface - for example caused by previously dried resistor prints - causes some departure from the thickness relations. But only if a cross-over or a thick film capacitor is very close to a resistor area an additional correction is considered necessary. A squeegee movement perpenticular to that used here let decrease square

\begin{tabular}{|c|c|c|c|c|c|c|c|c|c|c|}
\hline \multirow{2}{*}{\multicolumn{2}{|c|}{\begin{tabular}{l}
\multicolumn{1}{c}{ Paste } \\
$\begin{array}{l}\text { Parameter } \\
\text { [unit] }\end{array}$
\end{tabular}}} & \multicolumn{3}{|c|}{1311} & \multicolumn{3}{|c|}{1331} & \multicolumn{3}{|c|}{1361} \\
\hline & & $\begin{array}{l}R_{2}^{R}(L, 1) \\
{[\Omega / \square]}\end{array}$ & $\begin{array}{c}m_{2}(L) \\
-\end{array}$ & $\begin{array}{c}a(L) \\
-\end{array}$ & $\begin{array}{l}R_{2}^{0}(L, 1) \\
{[k \Omega / \square]}\end{array}$ & $\begin{array}{c}m_{2}(L) \\
-\end{array}$ & $\begin{array}{c}a(L) \\
-\end{array}$ & $\begin{array}{l}R_{2}^{D}(L, 1) \\
{[M \Omega / \square]}\end{array}$ & $\begin{array}{c}m_{2}(L) \\
-\end{array}$ & $\begin{array}{r}a(L) \\
-\end{array}$ \\
\hline \multirow{6}{*}{$\begin{array}{c}\text { length } \\
L \\
{[\mathrm{~mm}]}\end{array}$} & 0.5 & 16.8 & 1.10 & 0.05 & 0.85 & 1.095 & 0.065 & 0.55 & 0.975 & -0.04 \\
\hline & 1 & 13.3 & 1.06 & 0.045 & 0.95 & 1.08 & 0.04 & 1.03 & 0.91 & 0.01 \\
\hline & 1.5 & 14.0 & 1.12 & 0.07 & 1.09 & 1.11 & 0.05 & 1.24 & 1.00 & 0.03 \\
\hline & 2.5 & 13.8 & 1.12 & 0.10 & 1.29 & 1.10 & 0.04 & 1.25 & 1.04 & 0.04 \\
\hline & 4 & 13.9 & 1.135 & 0.065 & 1.42 & 1.085 & 0.025 & 1.23 & 1.15 & 0.07 \\
\hline & 7 & 14.6 & 1.165 & 0.09 & 1.61 & 1.12 & 0.03 & 1.26 & 1.15 & 0.08 \\
\hline
\end{tabular}
resistance particularly for long resistors. For lengths from 4 to $7 \mathrm{~mm}$ the decrease in square resistance will

TABLE I

The paste parameters $R^{\square}(L, 1), m(L), a(L)$ in the 2. approximation for the pastes 1311 , 1331 and 1361. 


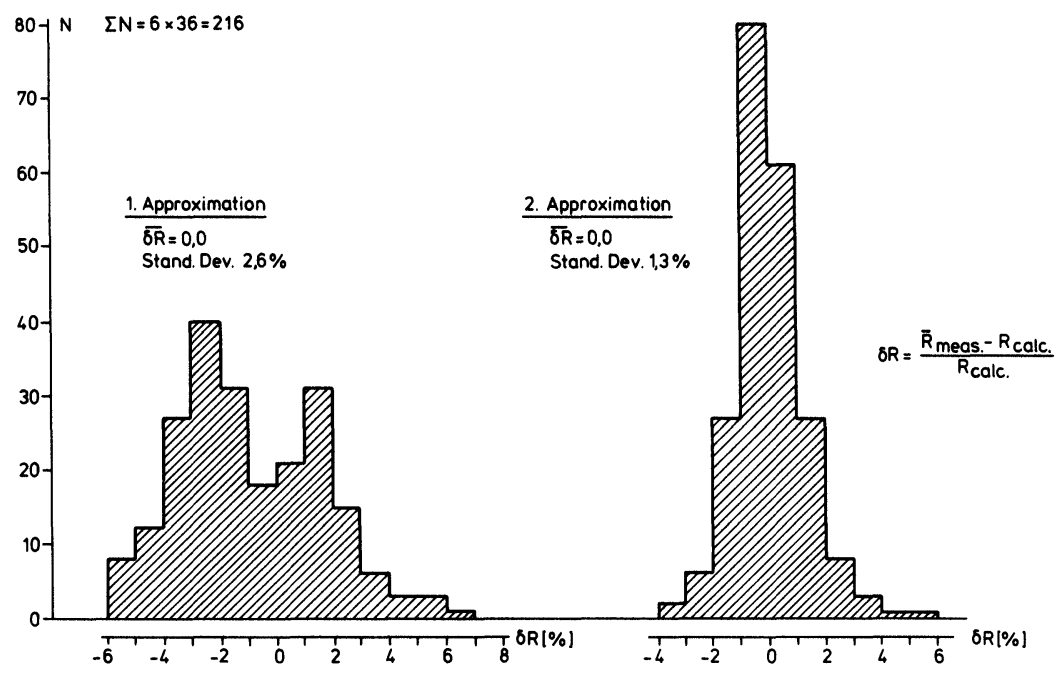

FIGURE 4 Error distribution between measured mean resistance values $\bar{R}$ and the values $R$ calculated from Eqs. (4) or (5) for all 6 pastes of the Du Pont 1300 series $(10 \Omega / \square$ to $1 \mathrm{M} \Omega / \square)$ for every the 36 geometries of the test pattern $\left(0.5 \times 0.5 \mathrm{~mm}^{2}\right.$ to $\left.7 \times 4 \mathrm{~mm}^{2}\right)$.

be about 5 to 10 percent. If this influence shall be taken into account another set of test circuits must be produced and evaluated.

\section{DIAGRAMS OF SQUARE RESISTANCE VALUES $R^{\square}(L, W)$}

If the computer system used includes an $x / y$ plotter or a CRT display, the square resistance can be displayed as a function of length $L$ for any required width $W$ immediately after computation of the paste parameters $R^{\square}(L, 1), m(L)$ and $a(L)$. The equations required are obtained by substituting the equations (4) or (5) found for the resistance $R$ in the equation $R^{\square}(L, W)=R \cdot W / L$ :

$$
\begin{aligned}
& R_{1}^{\square}(L, W)=R_{1}^{\square}(L, 1) \cdot W^{1-m_{1}} \\
& R_{2}^{\square}(L, W)=R_{2}^{\square}(L, 1) \cdot W^{1-m_{2}+a \cdot \ln W / 2}
\end{aligned}
$$

Figures 5 to 7 show such diagrams for pastes 1311, 1331 and 1361.

As $W$ is a independent variable in Eqs. (8) and (9), curves could be computed in close steps for any required value of $W$. This representation is extremely useful, as it shows at a glance what relationships will be present in various combinations of conductors and resistors. If the printing or firing conditions are changed, these diagrams show at a glance the effects on square resistance. The $R^{\square}(L, W)$ diagrams can thus play an important part in process control.

The form of the curves for the low-ohmic paste 1311 in Figure 5 shows that no diffusion influence

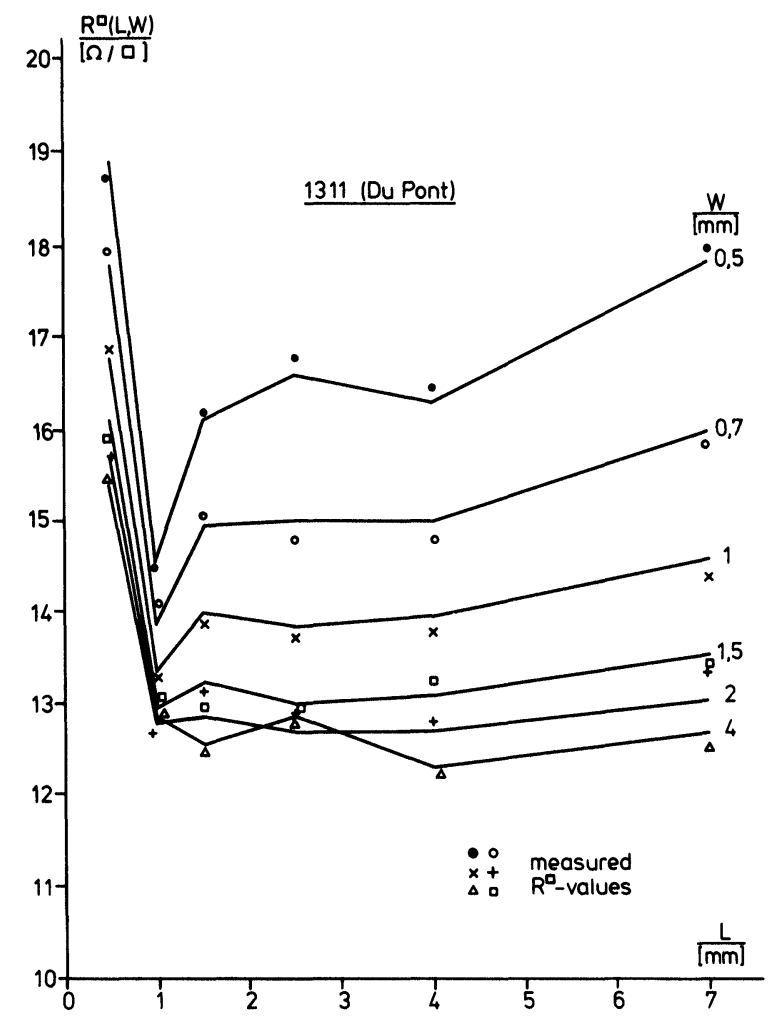

FIGURE 5 Calculated square resistance curves as a function of resistor length $L$ (Parameter: width $W$ ). 


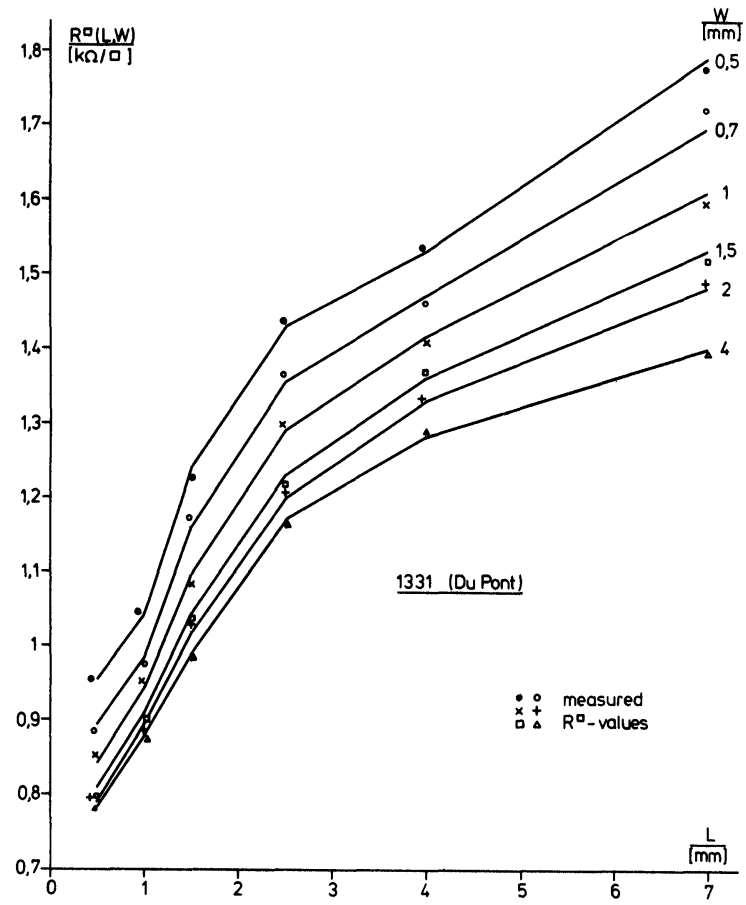

FIGURE 6 Calculated square resistance curves as a function of resistor length $L$ (Parameter: width $W$ ).

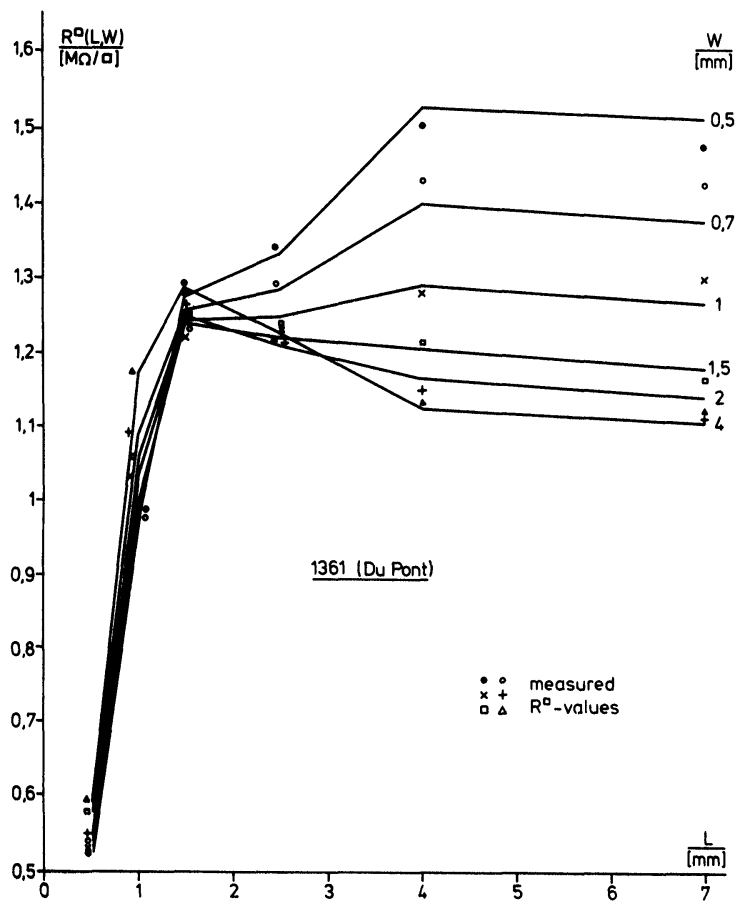

FIGURE 7 Calculated square resistance curves as a function of resistor length $L$ (Parameter: width $W$ ). can be found in long resistors. The drop in resistance for short resistors is over-compensated by an opposite effect; microscopic examination of the resistance films showed micro-cracks particularly in the neighbourhood of the conductor contacts. This increases the apparent square resistance, particularly for resistors with low $L / W$ ratio. Paste 1331 in Figure 6 shows a clear influence of diffusion on the form of the curve, square resistance dropping for long resistors also. This behaviour is qualitatively the same for all pastes of the Du Pont 1300 series from $R^{\square}=100 \Omega / \square$ to $R^{\square}=100 \mathrm{k} \Omega / \square$, but high-ohmic pastes show a greater slope. The behaviour of the $1 \mathrm{M} \Omega / \square$-paste 1361 (Figure 7) is surprising; the square resistance does not fall off for broad resistors, but it does suddenly drop for lengths less than $1.5 \mathrm{~mm}$. Obviously this paste is not merely a version of paste 1351 containing more glass, a conclusion which is also confirmed by examination of the surface structure.

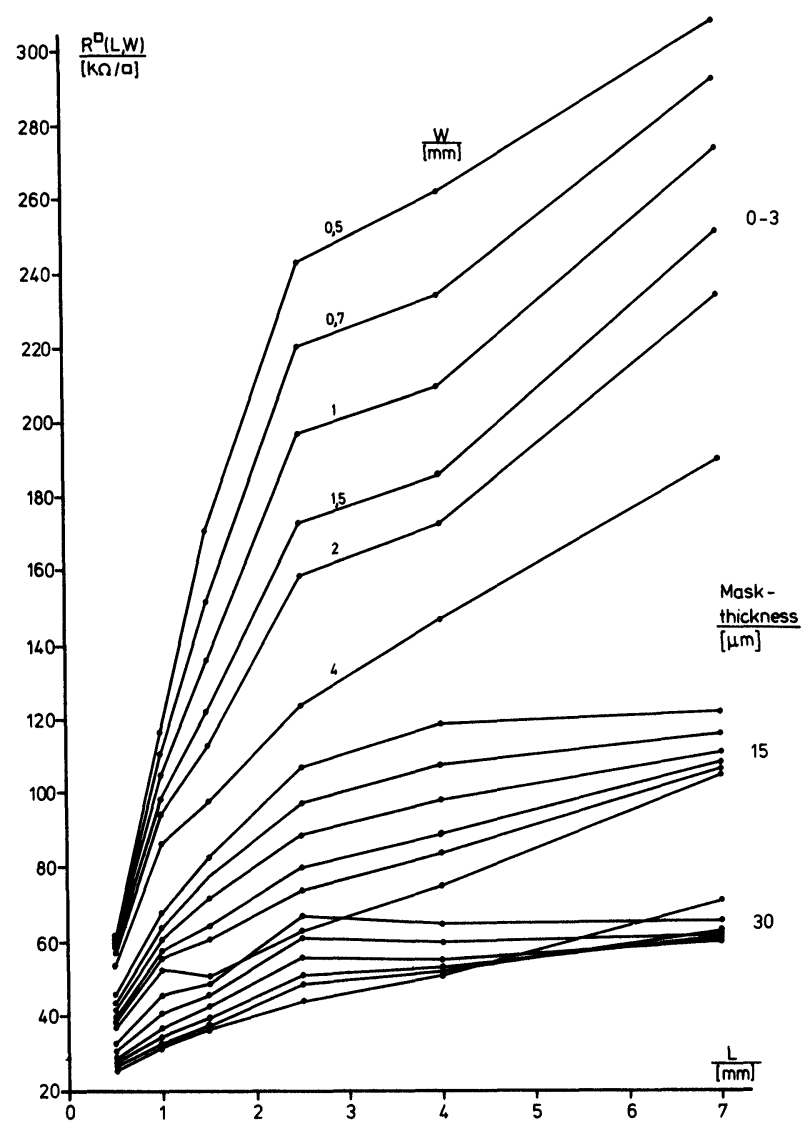

FIGURE 8 Calculated square resistance curves as a function of resistor length $L$ (Parameter: width $W$ ) for different mask thickness. 
The increase of square resistance which is always observed in narrow resistors in these pastes may be caused by the decrease in film thickness. We have however found complicated relationships between width and square resistance in some cases, especially with high-ohmic pastes. Although the square resistance of narrow resistors was mostly higher than that of broad resistors, the quantitative difference may be small. In some cases, even the opposite effect, a drop in square resistance with narrow resistors, can be observed. This is particularly the case when high-ohmic pastes are fired at high temperatures. The reasons for this phenomenon are still obscure. The strength of the effect of screen printing conditions on the form of the curves of $R^{\square}(L, W)$ is shown in Figure 8, the condition involved here being mask thickness. The prints were made with a directly-coated screen and mask thickness of $0-3,15$ and $30 \mu \mathrm{m}$ above the screen. The saw-tooth structure of the edges, indicated here for mask thickness zero (which is often observed in screen printing), was obviously the cause of the sharp rise in $R$ for narrow resistors. The larger drop in square resistance for short resistors can be contributed to the larger increase of paste thickness in short resistors compared to that in long resistors (with absence of mask thickness) and the fact, that silver diffusion occurs in the hole cross-section of the resistor, if its thickness is low. All three groups in Figure 8 were fired together and have the same palladium-silver contacts.

\section{ACKNOWLEDGEMENT}

I wish to thank Dipl. Phys. W. Schynoll for stimulating discussions and preparing the computer programmes.

\section{REFERENCES}

1. L. F. Miller, "Paste transfer in the screening process," Solid State Techn. (June 1969), pp. 46-52.

2. W. G. Dryden, "Achieving consistent results with thickfilm," Solid State Techn. (Sept. 1971), pp. 54-57.

3. C. Y. Kuo, "The contact resistance in thickfilm resistors," Proc. 1969 Hybrid Microelectr. Symp., pp. 263-269.

4. J. P. Garvin, S. J. Stein, "The influence of geometry and conductive terminations on thickfilm resistors," Proc. IEEE Comp. Conf., (1970), pp. 190-200.

5. L. A. Harper (Editor), Handbook of Thickfilm Hybrid Microelectronics, McGraw-Hill (1974), pp. 6.22-6.25.

6. J. A. Loughran, R. A. Sigsbee, "Termination anomalies in thickfilm resistors," Proc. 1969 Hybrid Microelectr. Symp., pp. 271-280.

7. C. J. Peckinpaugh, W. G. Proffitt, "Termination interface reaction with non-palladium resistors and its effect on apparent sheet resistivity," Proc. IEEE Comp. Conf. (1970), pp. 520-530.

8. J. A. van Hise, "Resistor interface and geometry phenomena," Proc. NEPCON East/West (1972), pp. 177-184.

9. D. E. Riemer, "The effect of geometry on the characteristics of thickfilm resistors," Proc. IEEE Comp. Conf. (1970), pp. 102-108.

10. C. F. Jefferson, "Geometry dependence of thickfilm resistors," Proc. IEEE Comp. Conf. (1970), pp. 209-215. 

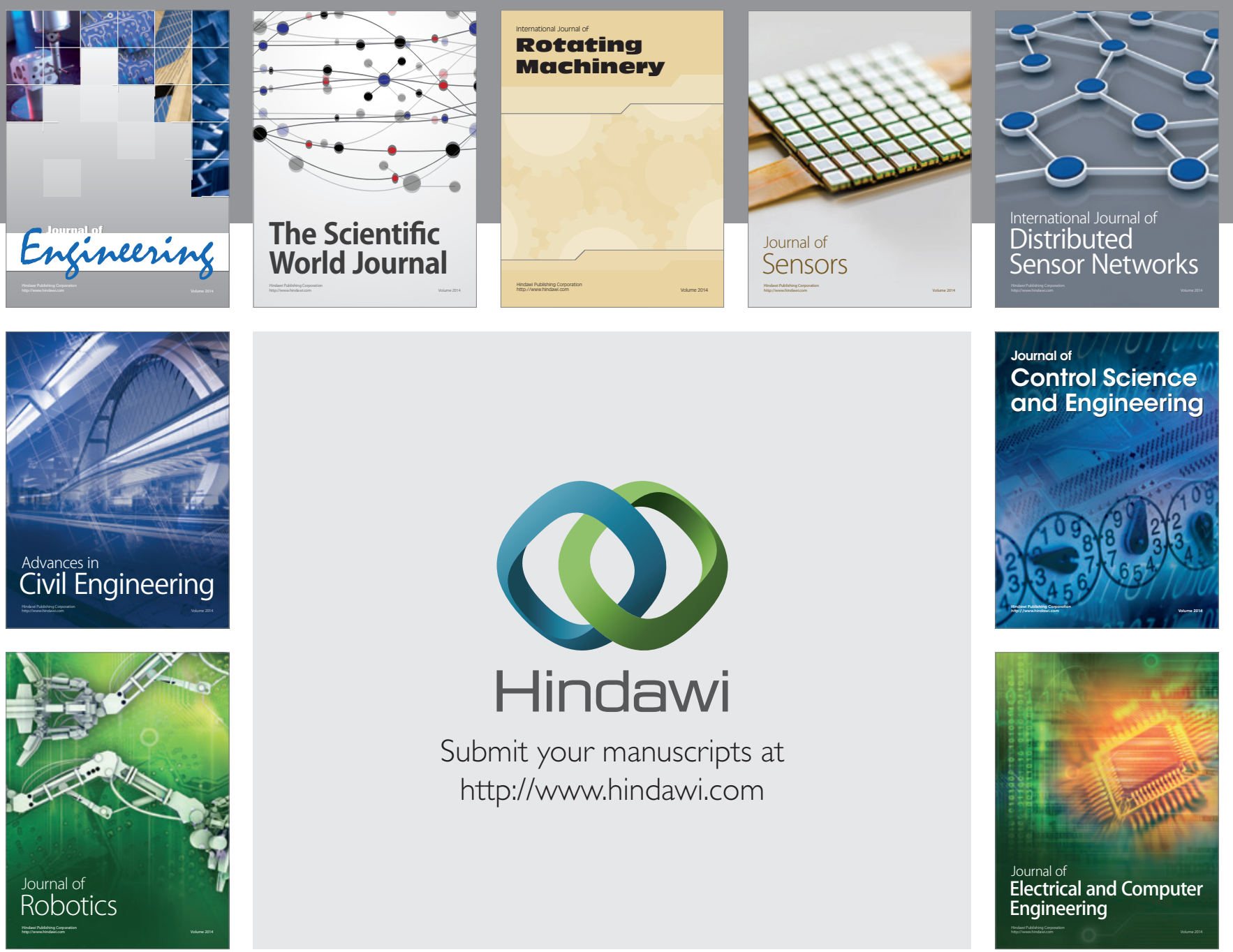

Submit your manuscripts at

http://www.hindawi.com
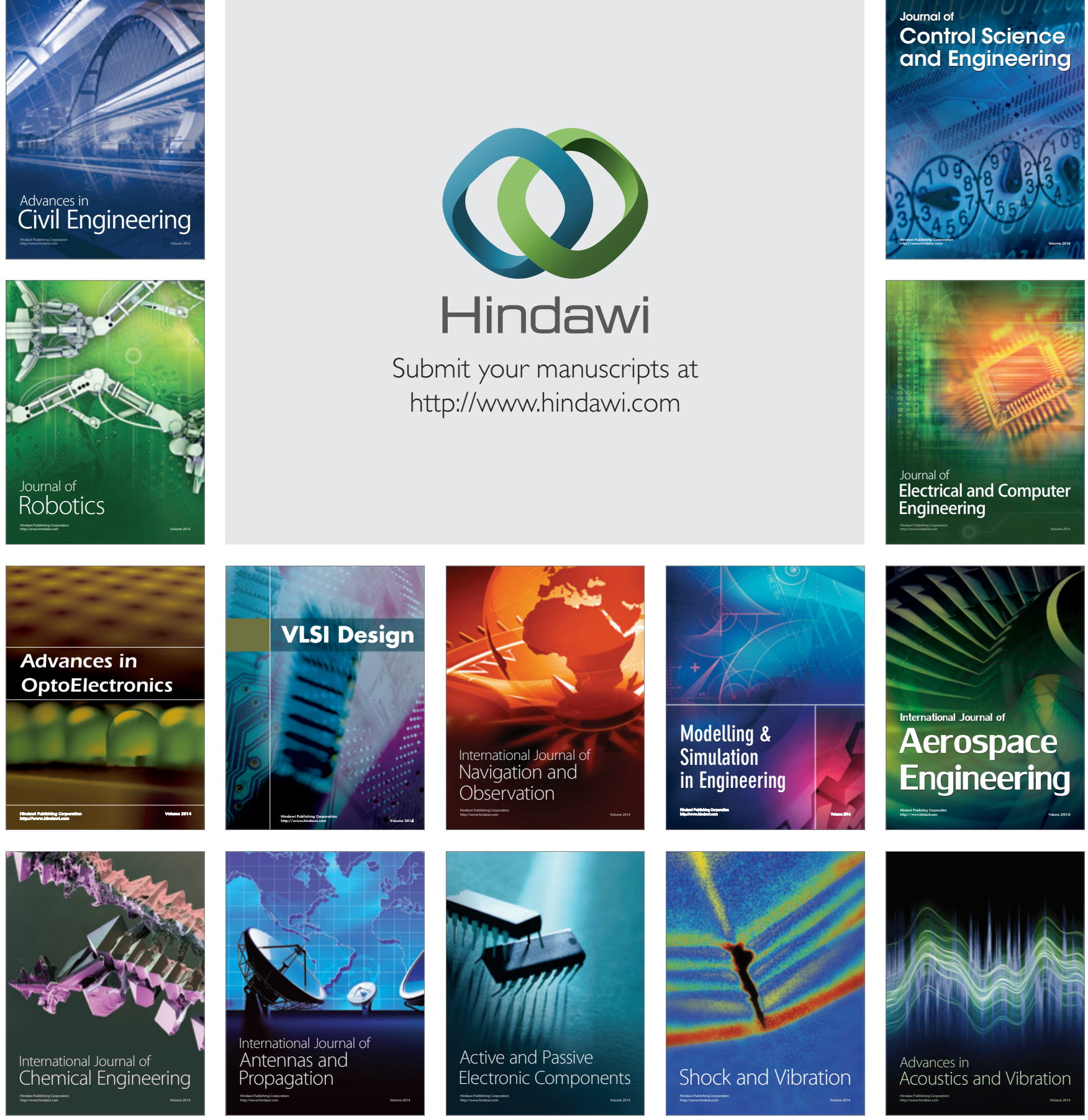